ЖЕЛЧНОКАМЕННАЯ БОЛЕЗНЬ КАК КЛИНИЧЕСКИЙ МАРКЕР МЕТАБОЛИЧЕСКОГО СИНДРОМА

\author{
( А.А. Свистунов ${ }^{1}$, М.А. Осадчук*, Н.В. Киреева', А.М. Осадчук²
}

'ФГАОУ ВО «Первый Московский государственный медицинский университет им. И.М. Сеченова» Министерства здравоохранения Российской Федерации (Сеченовский Университет), Москва, Россия

${ }^{2}$ ФГБОУ ВО «Самарский государственный медицинский университет» Министерства здравоохранения Российской Федерации, Самара, Россия

Распространенность желчнокаменной болезни, ее тесная патогенетическая связь с метаболическим синдромом, высокая частота хирургического вмешательства, значительные экономические потери выдвигают данную коморбидную патологию в ряд ведущих проблем современной клинической медицины. Факторы, ассоциированные с метаболическим синдромом, не только повышают риск развития желчнокаменной болезни, но и формируют основу немедикаментозной и медикаментозной терапии. Метаболический синдром часто определяет возникновение трех распространенных и потенциально опасных для жизни осложнений холелитиаза: острый холецистит, острый холангит и билиарный панкреатит. Поэтому решение данной проблемы связано с необходимостью раннего выявления дополнительных факторов риска холелитиаза, оптимизации ранней диагностической и прогностической модели существующей полиорганной патологии с целью уменьшения прогрессирования заболевания и его осложнений. Полученные в последние годы данные по геному человека с метаболическим синдромом и желчнокаменной болезнью дают возможность прогнозировать развитие коморбидной патологии и в полной мере обеспечивать результативность первичной профилактики.

КЛЮЧЕВЫЕ СЛОВА: желчнокаменная болезнь, холелитиаз, метаболический синдром, генетика, ожирение, ишемическая болезнь сердца, артериальная гипертензия, профилактика.

\title{
GALLSTONE DISEASE AS A CLINICAL MARKER OF METABOLIC SYNDROME
}

(c) Andrey A. Svistunov ${ }^{1}$, Mihail A. Osadchuk ${ }^{1 *}$, Natalia V. Kireeva ${ }^{1}$, Alexey M. Osadchuk².

'I.M. Sechenov First Moscow State Medical University (Sechenov University), Moscow, Russia

${ }^{2}$ Samara State Medical University, Samara, Russia.

The prevalence of cholelithiasis, its close pathogenetic connection with metabolic syndrome, high frequency of surgical intervention, significant economic losses put forward this comorbid pathology in a number of leading problems of modern clinical medicine. The factors associated with the metabolic syndrome not only increase the risk of developing cholelithiasis, but also form the basis of non-drug and drug therapy. Metabolic syndrome often determines the occurrence of three common and potentially life-threatening complications of cholelithiasis: acute cholecystitis, acute cholangitis and biliary pancreatitis. Therefore, the solution of this problem is associated with the need for early detection of additional risk factors for cholelithiasis, optimization of the early diagnostic and prognostic model of existing multi-organ pathology with the aim of reducing the progression of the disease and its complications. The data obtained in recent years on the human genome with metabolic syndrome and cholelithiasis make it possible to predict the development of comorbid pathology and to fully ensure the effectiveness of primary prevention.

KEYWORDS: Gallstones, cholelithiasis, metabolic syndrome, genetics, abdominal obesity, ischemic heart disease, hypertension, prevention.

\section{ВВЕДЕНИЕ}

В современном понимании метаболический синдром (МС) - это группа признаков и симптомов, которые способны увеличивать риск развития сердечно-сосудистых заболеваний и сахарного диабета (СД). В классическом варианте МС включает в себя центральное ожирение, артериальную гипертензию, гипергликемию и дислипидемию. В связи с этим основной проблемой данного синдромокомплекса является вопрос: способен ли он в совокупности повысить риск развития осложнений в большей степени, чем каждая отдельно взятая патология, входящая в состав МС или ассоциированная с ним [1]. Это тем более важно, что рамки МС постоянно расширяются за счет включения неалкогольной жировой болезни печени (НЖБП) и желчнокаменной болезни (ЖКБ), которые приобретают характер эпидемии с высоким уровнем осложнений и потребностью в оперативном вмешательстве $[2,3]$. В связи с этим целью настоящего обзора явилась необходимость расширить имеющиеся сведения о связи МС с ЖКБ и определить основные этиопатогенетические и прогностические критерии данного сочетания, основанные на совокупности данных анализа литературы, включающей как мета-анализы, так и систематические обзоры и оригинальные исследования. 


\section{ЭПИДЕМИОЛОГИЯ ХОЛЕЛИТИАЗА ПРИ МЕТАБОЛИЧЕСКОМ СИНДРОМЕ}

ЖКБ по распространенности, частоте оперативного вмешательства и экономическим потерям относится к одной из самых затратных областей мирового здравоохранения [4]. Данной патологией страдают от $10 \%$ до $20 \%$ населения европейских стран, при этом в последние годы она приобрела устойчивую тенденцию к росту. Так, у 15-20\% населения Германии верифицируются конкременты в желчном пузыре (ЖП), и ежегодно в этой стране проводится более 190 тыс. холецистэктомий [5]. В США у 6-7,4\% мужчин и 9\% женщин определяются конкременты в ЖП [6]. Самая высокая распространенность холелитиаза регистрируется у коренных американцев. В последнее десятилетие распространенность ЖКБ в Азии и Африке приближается к европейским и североамериканским показателям. При этом следует подчеркнуть, что частота ЖКБ в Китае составляет 10,8\% и у $2,97 \%$ из них заканчивается холецистэктомией [7]. Повсеместно наблюдающаяся тенденция к увеличению числа людей с избыточной массой тела, достигшая порога эпидемии [8], способствует увеличению заболеваемости холелитиазом [9]. Так, проведенное в Тайване перекрестное исследование в течение 2011-2012 гг., охватывающее 12050 человек, свидетельствует о том, что частота МС и ЖКБ составила соответственно 27,20\% и 6,16\%. При этом МС с высокой степенью достоверности ассоциировался с увеличением риска ЖКБ (отношение шансов (ОШ) =1,61; 95\% доверительный интервал (ДИ) $=1,366-1,898 ; \mathrm{P}<0,001)$. В датском когортном исследовании с систематическим мета-анализом было подтверждено, что возраст, женский пол, индекс массы тела (ИМТ), высокий уровень холестерина, липопротеидов низкой плотности (ЛПНП) и полипы являются независимыми детерминантами образования конкремента в ЖП [10]. Количество больных с ЖКБ увеличивается и в стареющей популяции с высокой распространенностью в ней МС и ожирения [11].

\section{ОБЩИЕ ЭТИОЛОГИЧЕСКИЕ И ПАТОГЕНЕТИЧЕСКИЕ ФАКТОРЫ РАЗВИТИЯ ХОЛЕЛИТИАЗА ПРИ МС}

Данные нескольких крупных эпидемиологических исследований в США, Европе, Китае и Японии свидетельствуют о том, что длительный период избыточного питания с доминированием рафинированных углеводов при наличии гипертриглицеридемии на фоне дефицита пищевых волокон выступает провоцирующим фактором этиологического риска холелитиаза [12]. Ожирение является фактором риска для образования конкрементов и дислипидемии и подвергает пациентов повышенному риску осложнений ЖКБ. Вместе с тем быстрая потеря веса, достигнутая с помощью низкокалорийных диет или бариатрической хирургии, также является фактором риска холелитиаза у пациентов с первоначальным ожирением.

Основные патогенетические факторы для образования холестериновых камней, ведущие к осаждению кристаллов холестерина, формированию гипокинезии ЖП и конкрементов включают генетический фон, гиперсекрецию печенью холестерина и перенасыщенную желчь [4]. При этом процесс холелитиаза коррелиру- ет с резистентностью к инсулину, ожирением, МС и СД 2 типа. В последние годы активно изучается роль ядерных рецепторов сигнальных путей, микробиоты кишечника и эпигенома в манифестации и прогрессировании холелитиаза $[4,13,14]$.

Инициирующим фактором холелитиаза могут выступать генетические факторы. Крупное исследование в рамках Шведского двойного реестра дало убедительные доказательства роли генома в патогенезе ЖКБ [15]. Основываясь на данных 43141 близнецовых пар, авторы доказали, что генетические факторы составляют 25\% (95\% ДИ 9-40\%) фенотипической вариации среди близнецов [16]. Аналогичный анализ в 358 семьях, проведенный в Висконсине, который охватывал по крайней мере двоих страдающих ожирением бразильских детей в данных семьях, определил, что наследуемость по холелитиазу составляет $29 \pm 14 \%$ [17].

В первых опубликованных результатах исследований по определению генома в большой когорте пациентов с ЖКБ из Германии [18], затрагивающих и сиб-пары [19], был обнаружен общий вариант гена (p.D19H) переносчика холестерина гепатоканала $A B C G 5$, и $A B G 8$, который выступает генетическим фактором риска для образования конкрементов в ЖП [18]. Гепатоциты экспрессируют специфические транспортные белки для липидов желчи - известные как АТФ-связывающие кассеты (ABC) транспортеры. Транспортер $A B C B 11$ представляет собой насос для экспорта желчных солей, $A B C B 4$ является транспортером для фосфатидилхолина, а $A B C G 5 / A B C G 8$ образуют облигатные гетеродимеры, участвующие в секреции холестерина желчи $[19,20]$. Проведенные исследования свидетельствуют о том, что вариант p.D19H определяет коэффициенты шансов манифестации ЖКБ, соответственно 2-3\% и 7\% для гетерозиготных и гомозиготных носителей, и 8-11\% пациентов с данной патологией могут быть отнесены к этому варианту [18]. Общие мутации в транспортере холестерина печени $A B C G 8$ дают большую часть генетического риска развития конкрементов, что составляет порядка $25 \%$ общей популяции (рис. 1) [21]. В ряде работ доказано, что носители литогенного варианта $A B G G 819 H$ демонстрируют более низкие уровни стерола в сыворотке крови и более высокое содержание предшественников холестерина [20, 22], что указывает на снижение абсорбции холестерина и активацию его биосинтеза. Это может быть клинически значимым событием, поскольку ингибиторы HMG-CoA-редуктазы могут быть особенно эффективными в снижении уровня холестерина сыворотки крови [11, 22, 23]. Заслуживают внимания данные о том, что ИБС и ЖКБ представляют собой сочетанную патологию, ассоциированную с несколькими генетическими факторами, включая полиморфизм в генах, кодирующих аполипопротеин E [24] и АТФ-связывающие кассеты G5 и G8 [25].

До настоящего времени не утихает дискуссия о роли бактериальной инфекции в генезе холелитиаза. С этих позиций заслуживает внимания работа T. Matyjas и coавт. [21], касающаяся наличия и идентификации бактериальных штаммов, обнаруженныхв желчи и конкрементах, расположенных в ЖП и желчных протоках. Бактериальную культуру оценивали у 92 пациентов. Культура желчи была положительной у 46 пациентов, что составляет $50 \%$ от исследуемой группы. У данного контингента 
Соли желчных кислот



Рис. 1. Схематическое представление наиболее важных транспортных процессов в канальной мембране гепатоцитов, опосредованных транспортными средствами АВС.

Na-3TT - натрий-зависимый транспортер таурохолата

ПЭЖК - помпа, экскретирующая желчные кислоты

$\mathrm{BCRP}$ - белок устойчивости к раку молочной железы

$A B C G 5, A B C G 8$ - гены, кодирующие белки стеролин-1 и стеролин-2

ABCC2 (MRP2), ABCG2 (BCRP), ABCB1 (MDR1 Pgp) - АTФ-связывающий

кассетный транспортер

$\mathrm{AQP}$ - аквапорин

больных были выращены следующие штаммы бактерий: Enterococcus spp. (44\%), Escherichia coli (37\%) и Klebsiella spp. (35\%). Кандидоз, сопровождающийся бактериальной инфекцией, был обнаружен у 7 пациентов (15\%). Молекулярное исследование желчных камней выявило ДНК Enterococcus spp., Escherichia spp., Streptococcus spp. и Clostridium spp., а в культуре желчи Enterococcus spp. (avium и faecalis).

Определенный интерес представляют работы по определению роли инфекции Helicobacter pylori (H. pylori) при ЖКБ, тем более что данный микроорганизм может выступать предиктором развития МС и НЖБП [26], а успешно проведенная эрадикация уменьшает такие проявления MC, как увеличение окружности талии, повышенный уровень глюкозы в крови, и улучшает показатели липидного профиля [27]. Патофизиология микробного влияния Н. pylori на МС связана с несколькими возможными механизмами, включая индукцию провоспалительных цитокинов, которые влияют на липидный и гормональный профиль, что способствует прогрессированию патологического процесса с формированием фиброза печени и резистентности к инсулину [28]. L. Cen и соавт. [29] приводят данные мета-анализа по определению связи инфекции Н. pylori ЖП с хроническим холециститом и ЖКБ. Авторы опирались на базы данных PubMed, EMBASE и Cochrane для идентификации всех исследований, опубликованных до августа 2017 г. Объединенные ОШ и соответствующие 95\% ДИ были получены с использованием модели случайных эффектов. Был также проведен стратифицированный анализ на гетерогенность и чувствительность. С этой целью было отобрано 18 исследований с участием 1544 участников и 1061 больных с хроническим холециститом или ЖКБ. Н. pylori инфекция ЖП была значительно связана с повышенным риском хронического холецистита и холелитиаза (ОШ=3,022, 95\% ДИ, 1,897-4,815, 12=20,1\%). Этот метаанализ показывает, что Н. pylori инфекция ЖП ассоциируется с риском развития хронического холецистита и ЖКБ.
Микробиота кишечника также играет существенную роль в патогенезе МС [30]. Нарушение баланса между кишечной микрофлорой и иммунной системой макроорганизма может привести к кишечной транслокации бактериальных фрагментов и развитию «метаболического эндотоксикоза», вызванного бактериями и/или бактериальными фрагментами, такими как липополисахариды, которые проходят через кишечный барьер в кровь, что приводит к системному воспалению. Присутствие представителей кишечной микробиоты в литогенной желчи [31] может быть признаком повышенной кишечной проницаемости при билиарной обструкции [32], что способствует манифестации воспалительной реакции и образованию камня [8]. Метаболический эндотоксикоз может стимулировать инфильтрацию макрофагов и активацию синтеза провоспалительных цитокинов, увеличивать продукцию сигнальных цитокинов и ингибировать белковый синтез. Современные представления предполагают, что возникшее хроническое системное воспаление способствует развитию резистентности к инсулину, СД, ожирению и ЖКБ. Так, уменьшение числа Bacteroides и увеличение численности Firmicutes обычно связано с MC $[4,13,14]$. Первичные желчные кислоты в кишечнике не только регулируют переваривание и абсорбцию холестерина, триглицеридов и жирорастворимых витаминов, но также играют ключевую роль в качестве сигнальных молекул при модулировании пролиферации эпителиальных клеток, экспрессии генов, метаболизме липидов и глюкозы путем активации фарнезоид X рецептора (FXR) и рецептора желчной кислоты-1, связанного с G-белком (GPBAR-1, также известный как TGR5) в ткани печени, кишечника, мышц и жировой ткани. FXR и GPBAR-1 участвуют в биосинтезе и энтерогепатической циркуляции первичных желчных кислот и, в качестве сигнальных молекул, при метаболизме липидов и глюкозы [33].

Следовательно, ЖКБ, вероятно, является результатом сложного взаимодействия окружающей среды, генетических факторов, высокоуглеводных, высокожирных и низкокалорийных диет [34]. Эта гипотеза подтверждается значительным повышением уровня распространенности холестерина в желчных камнях у коренных американцев и в современных городских центрах Восточной Азии, что связано с введением высококалорийных «западных» диет [35].

\section{СВЯЗЬ ХОЛЕЛИТИАЗА С ОСНОВНЫМИ КОМПОНЕНТАМИ МС}

Заслуживают внимания данные о том, что при атеросклерозе риск заболевания ЖКБ увеличивается при наличии ожирения, СД 2 типа, резистентности к инсулину и дислипидемии. Все эти составляющие смело можно отнести к МС, при котором ЖКБ считается еще одним «попутчиком» $[36,37]$. Шанс развития острого панкреатита и тяжелого течения ЖКБ выше у пациентов с ожирением из-за специфических патогенных факторов, включая перенасыщение желчи и ее кристаллизацию, быструю потерю веса и висцеральное ожирение. Политика здравоохранения, направленная на снижение заболеваемости ожирением во всем мире, уменьшает заболеваемость ЖКБ и ее осложнения. 
В ряде работ продемонстрирована тесная взаимозависимость между наличием конкрементов в ЖП, дислипидемией, МС, сердечно-сосудистыми заболеваниями. В частности, было доказано, что артериальная жесткость сосудов является не только показателем субклинического атеросклероза, но и важным прогностическим фактором, провоцирующим развитие ЖКБ [38]. Ишемическая болезнь сердца (ИБС) и ЖКБ являются следствием накопления холестерина соответственно в стенке коронарной артерии и полости ЖП. Эти два заболевания имеют несколько общих факторов риска, включая возраст, пол, избыточный вес и нарушения метаболизма липидов и глюкозы [39-42]. Указанные факторы также являются ключевыми компонентами для диагностики МС [43]. МС тесно ассоциируется с атеросклерозом коронарных артерий (АКА) [39], а ЖКБ можно рассматривать как составляющую МС. Кроме того, проведенные исследования в этой области подчеркивают частую ассоциацию ЖКБ, НЖБП и ИБС [6].

Заслуживает внимания клиническое исследование, проведенное в Шанхае в клинике сердечно-сосудистой хирургии с января 2007 г. по сентябрь 2011 г. [7]. В этом кросс-секционном исследовании впервые подверглись анализу пациенты с ЖКБ и МС, у которых диагноз АКА был поставлен на основании данных коронарной ангиографии. На основании проведенного исследования авторы выявили значительную ассоциацию между ЖКБ и АКА (скорректированный OR 1,59; 95\% ДИ 1,10-2,31), причем эта связь подтверждена анализом парных случаев (скорректированный OR 1,69; 95\% ДИ 1,08-2,65). В работе была продемонстрирована корреляция между ЖКБ и АКА, о чем свидетельствует значительно более высокая распространенность ЖКБ у пациентов с данной патологией (19,5\% против 11,3\% в группе сравнения, $p<0,01)$.

Известно, что пациенты с ИБС и ЖКБ имеют несколько общих факторов риска, таких как более высокий уровень холестерина ЛПНП и более низкий уровень холестерина липопротеидов высокой плотности (ЛПВП). Они связаны с АКА даже после сопоставления по возрасту, полу и МС. Однотипность липидного профиля при ИБС и ЖКБ может быть объяснена общими путями метаболизма холестерина по оси кишечник - печень [44]. Интересно, что холестерин ЛПВП обратимо связан с обоими заболеваниями, так как он играет роль в обратном транспорте холестерина [45] и поглощается печеночным рецептором В типа I (SRBI). Повышенная экспрессия печеночной SRBI обнаружена у китайских пациентов с ЖКБ [46], что может служить источником чрезмерного поступления холестерина в желчь $[47,48]$. Повышенный уровень глюкозы крови натощак является другим фактором, связанным с АКА и ЖКБ [3]. Наличие при этом МС может влиять на метаболизм холестерина и липопротеина и вызывать нарушение сократимости ЖП [49].

В ряде работ продемонстрирована ассоциация между метаболическими биомаркерами ожирения, включая резистентность к инсулину, сосудистую дисфункцию, системное воспаление, генетическую восприимчивость и ЖКБ или последующей холецистэктомией в популяционном кросс-секционном исследовании [50]. Всего было включено 2650 участников, из которых 422 - с ЖКБ. Ассоциации между выбранными метаболическими биомаркерами и ЖКБ оценивались с помощью многомерных моделей логистической регрессии и выражались как ОШ и 95\% ДИ. ЖКБ коррелировала с глюкозой натощак (ОШ 1,14; 95\% ДИ 1,05-1,24]), инсулином натощак (ОШ 1,03; 95\% ДИ 1,01-1,05), резистентностью к инсулину (ОШ 1,18; 95\% ДИ 1,02-1,36), МС (ОШ 1,51; 95\% ДИ 1,16-1,96), количеством лейкоцитов (ОШ 1,07; 95\% ДИ 1,00-1,15) и С-реактивным белком (ОШ 1,03; 95\% ДИ 1,01-1,05). Также была обнаружена тенденция к ассоциации с рецептором растворимого урокиназного плазминогена (ОШ 1,08; 95\% ДИ 0,99-1,18), MC4R (рецептор меланокортина 4) (rs17782313) (ОШ 1,27; 95\% ДИ 1,02-1,58), МАР2К5 (митоген-активированная протеинкиназа-киназа 5) (rs2241423) (ОШ 1,80; 95\% ДИ 1,04-3,41), NRXN3 (нейрексин-3-альфа представляет собой белок, который у человека кодируется геном NRXN3) (rs10146997) (ОШ 1,26; 95\% ДИ 1,01-1,57), HНЕX (гомеобокссодержащий ген) (rs1111875) (ОШ 1,29; 95\% ДИ 1,03-1,62), FAIM2 (фазовая апоптотическая ингибирующая молекула 2) (rs7138803) (ОШ 0,66; 95\% ДИ 0,48-0,91) и аполипопротеин Е4 аллель (ОШ 0,76; 95 \% ДИ 0,59-0,98) были связаны с ЖКБ. Связь между ИМТ и ЖКБ объяснялась резистентностью к инсулину. Таким образом, резистентность к инсулину, системное воспаление, генетическое ожирение или аллели риска развития СД 2 типа тесно связаны с ЖКБ [50].

\section{ХОЛЕЛИТИАЗ И ЕГО АССОЦИАЦИЯ С МС И ДРУГИМИ ЗАБОЛЕВАНИЯМИ ПИЩЕВАРИТЕЛЬНОГО ТРАКТА}

Недавно проведенные исследования показывают, что МС может играть определенную роль в манифестации ряда онкологических заболеваний. Так, холелитиаз, инфекция HBV и MC выступают потенциальными факторами риска развития рака желчного пузыря и желчевыводящих путей [51]. У пациентов с МС из-за наличия специфических патогенных факторов, включая перенасыщение желчи, ее кристаллизацию и висцеральное ожирение, шанс развития острого панкреатита и его тяжесть значительно увеличиваются.

ЖКБ и НЖБП часто сосуществуют вместе, и обе патологии тесно связаны с ожирением и резистентностью к инсулину. Печень является ключевым органом, который регулирует метаболизм холестерина и контролирует концентрацию холестерина в плазме крови и ЖП [44]. Данные пути метаболизма нарушаются у пациентов с МС, СД и НЖБП $[52,53]$, что приводит к дислипидемии или перенасыщению содержимого желчного пузыря холестерином. Связь между ЖКБ и НЖБП является сложной и двунаправленной. Несколько клинических исследований и систематических обзоров рассматривают связь между НЖБП и ЖКБ, подчеркивая, что НЖБП является независимым фактором риска для ЖКБ [54]. Вместе с тем ЖКБ также была признана независимым фактором риска и для НЖБП. Тесная патогенетическая связь ЖКБ, МС и НЖБП продемонстрирована и в других работах [55]. При этом авторы подчеркивают, что эти заболевания имеют сходные факторы риска, такие как ожирение, гипертриглицеридемия и СД [56, 57].

НЖБП, высокий ИМТ, женский пол, повышенный уровень ЛПНП, снижение содержания ЛПВП, использование контрацептивов, прием алкоголя, СД, гиподинамия, часто рожающие женщины, питьевая вода с избыточным содержанием железа и МС являются взаимоотягощаю- 
щими факторами, стимулирующими образование камней в ЖП и увеличивающими частоту осложнений [58].

\section{ЗАКЛЮЧЕНИЕ}

Наличие МС увеличивает частоту холелитиаза и манифестацию потенциально опасных для жизни осложнений ЖКБ (острый холецистит, острый холангит и билиарный панкреатит). Хотя современные знания о генетике и патофизиологии холелитиаза в последнее время расширились, современные алгоритмы лечения остаются преимущественно инвазивными и основаны на хирургическом вмешательстве [59]. Следовательно, будущие усилия здравоохранения должны быть сосредоточены на новых превентивных стратегиях для предупрежде- ния возникновения камнеобразования у пациентов как с MC, так и среди всего населения в целом. В этом плане большие надежды возлагаются на ранние доклинические критерии прогнозирования развития холелитиаза у больных с МС, основанные на генетических, микробиологических и лабораторных маркерах.

\section{ДОПОЛНИТЕЛЬНАЯ ИНФОРМАЦИЯ}

Конфликт интересов. Авторы декларируют отсутствие явных и потенциальных конфликтов интересов, связанных с публикацией настоящей статьи.

Участие авторов. Все авторы внесли значимый вклад в проведение поисково-аналитической работы и подготовку статьи, прочли и одобрили финальную версию перед публикацией.

\section{СПИСОК ЛИТЕРАТУРЫ | REFERENCES}

1. Hoffman EL, VonWald T, Hansen K. The metabolic syndrome. S. D. Med. 2015;Spec No:24-28.

2. Halmos T, Suba I. A nem alkoholos zsírmáj mint a metabolikus szindróma komponense és kauzális kapcsolatai egyéb kórképekkel. Orv. Hetil. 2017;158(52):2051-2061. doi: 10.1556/650.2017.30936.

3. Chen L-Y. Metabolic syndrome and gallstone disease. World J. Gastroenterol. 2012;18(31):4215. doi: 10.3748/wjg.v18.i31.4215.

4. Di Ciaula A, Wang DQH, Portincasa P. An update on the pathogenesis of cholesterol gallstone disease. Current Opinion in Gastroenterology. 2018;34(2):71-80. doi: 10.1097/mog.0000000000000423.

5. Lammert F, Neubrand M, Bittner R, et al. Kurzfassung der aktualisierten S3-Leitlinie der DGVS und DGVC zur Diagnostik und Behandlung von Gallensteinen. DMW - Deutsche Medizinische Wochenschrift. 2008;133(7):311-316. doi: 10.1055/s-2008-1046712

6. Ruhl CE, Everhart JE. Relationship of Non-alcoholic Fatty Liver Disease With Cholecystectomy in the US Population. The American Journal of Gastroenterology. 2013;108(6):952-958. doi: 10.1038/ajg.2013.70.

7. Schulz C, Jiang Z-Y, Sheng X, et al. Gallbladder Gallstone Disease Is Associated with Newly Diagnosed Coronary Artery Atherosclerotic Disease: A Cross-Sectional Study. PLoS One. 2013;8(9):e75400. doi: 10.1371/journal.pone.0075400.

8. Дедов И.И., Мельниченко Г.А., Романцова Т.И. Стратегия управления ожирением: итоги Всероссийской наблюдательной программы «ПримаВера». // Ожирение и метаболизм. - 2016 - Т.13. - №1. C. 36-44. [Dedov II, Mel'nichenko GA, Romantsova Tyl. The strategy of obesity management: the results of All-Russian observational program "Primavera". Obesity and metabolism. 2016;13(1):36-44. (In Russ).] doi: 10.14341/omet2016136-44.

9. Portincasa P, Di Ciaula A, de Bari O, et al. Management of gallstones and its related complications. Expert Review of Gastroenterology \& Hepatology. 2015;10(1):93-112. doi: 10.1586/17474124.2016.1109445.

10. Shabanzadeh DM, Sørensen LT, Jørgensen T. Determinants for gallstone formation - a new data cohort study and a systematic review with meta-analysis. Scand. J. Gastroenterol. 2016;51 (10):1239-1248. doi: 10.1080/00365521.2016.1182583.

11. Lammert F, Miquel J-F. Gallstone disease: From genes to evidence-based therapy. J. Hepatol. 2008;48:S124-S135. doi: 10.1016/j.jhep.2008.01.012.

12. Munnich A, Feingold J. Book Review: The Genetic Basis of Common Diseases (Oxford Monographs on Medical Genetics.). Second edition. Edited by King RA, Rotter JI, Motulsky AG.. 1076 pp., illustrated. New York, Oxford University. N. Engl. J. Med. 2003;349(4):410-410. doi: 10.1056/nejm200307243490423.

13. Ferrer M, Ruiz A, Lanza F, et al. Microbiota from the distal guts of lean and obese adolescents exhibit partial functional redundancy besides clear differences in community structure. Environ. Microbiol. 2013;15(1):211-226. doi: 10.1111/j.1462-2920.2012.02845.x.

14. He M, Shi B. Gut microbiota as a potential target of metabolic syndrome: the role of probiotics and prebiotics. Cell \& Bioscience. 2017;7(1). doi: 10.1186/s13578-017-0183-1.

15. Katsika D, Grjibovski A, Einarsson C, et al. Genetic and environmental influences on symptomatic gallstone disease: A Swedish study of 43,141 twin pairs. Hepatology. 2005;41(5):1138-1143. doi: 10.1002/hep.20654.
16. Lyons MA, Wittenburg H. Cholesterol Gallstone Susceptibility Loci: A Mouse Map, Candidate Gene Evaluation, and Guide to Human LITH Genes. Gastroenterology. 2006;131(6):1943-1970. doi: 10.1053/j.gastro.2006.10.024.

17. Nakeeb A, Comuzzie AG, Martin L, et al. Gallstones. Ann. Surg. 2002;235(6):842-849. doi: 10.1097/00000658-200206000-00012.

18. Grünhage F, Acalovschi M, Tirziu S, et al. Increased gallstone risk in humans conferred by common variant of hepatic ATP-binding cassette transporter for cholesterol. Hepatology. 2007;46(3):793-801. doi: $10.1002 /$ hep. 21847 .

19. Elferink RPJO, Paulusma CC, Groen AK. Hepatocanalicular Transport Defects: Pathophysiologic Mechanisms of Rare Diseases. Gastroenterology. 2006;130(3):908-925. doi: 10.1053/j.gastro.2005.08.052.

20. Acalovschi M, Ciocan A, Mostean O, et al. Are plasma lipid levels related to $A B C G 5 / A B C G 8$ polymorphisms? Eur. J. Intern. Med. 2006;17(7):490-494. doi: 10.1016/j.jjim.2006.04.012.

21. Matyjas T, Kaczka K, Witas H, et al. Cholelithiasis - always infected? Pol. Przegl. Chir. 2017;89(3):23-26.

22. Wittenburg H, Lyons MA, Li R, et al. FXR and ABCG5/ABCG8 as determinants of cholesterol gallstone formation from quantitative trait locus mapping in mice. Gastroenterology. 2003;125(3):868-881. doi: 10.1016/s0016-5085(03)01053-9.

23. Kajinami K, Brousseau ME, Nartsupha C, et al. ATP binding cassette transporter G5 and G8 genotypes and plasma lipoprotein levels before and after treatment with atorvastatin. J. Lipid Res. 2004;45(4):653-656. doi: 10.1194/jlr.M300278-JLR200.

24. Mandell MS, Xue P, Niu W-Q, et al. A Meta-Analysis of Apolipoprotein E Gene $\varepsilon 2 / \varepsilon 3 / \varepsilon 4$ Polymorphism for Gallbladder Stone Disease. PLoS One. 2012;7(9):e45849. doi: 10.1371/journal.pone.0045849.

25. Koeijvoets KCMC, van der Net JB, Dallinga-Thie GM, et al. ABCG8 gene polymorphisms, plasma cholesterol concentrations, and risk of cardiovascular disease in familial hypercholesterolemia. Atherosclerosis. 2009;204(2):453-458. doi: 10.1016/j.atherosclerosis.2008.09.018.

26. Kountouras J, Boziki M, Polyzos SA, et al. The Emerging Role of Helicobacter Pylori-Induced Metabolic Gastrointestinal Dysmotility and Neurodegeneration. Curr. Mol. Med. 2017;17(6):389-404 doi: 10.2174/1566524018666171219094837.

27. Mokhtare M, Mirfakhraee H, Arshad M, et al. The effects of helicobacter pylori eradication on modification of metabolic syndrome parameters in patients with functional dyspepsia. Diabetes Metab Syndr. 2017;11 Suppl 2:S1031-S1035. doi: 10.1016/j.dsx.2017.07.035.

28. Tang DM, Kumar S. The Association Between Helicobacter pylori Infection and Nonalcoholic Fatty Liver Disease. Current gastroenterology reports. 2017;19(2). doi: 10.1007/s11894-017-0545-1.

29. Cen L, Pan J, Zhou B, et al. Helicobacter Pylori infection of the gallbladder and the risk of chronic cholecystitis and cholelithiasis: A systematic review and meta-analysis. Helicobacter. 2018;23(1):e12457. doi: 10.1111/hel.12457

30. Pascale A, Marchesi N, Marelli C, et al. Microbiota and metabolic diseases. Endocrine. 2018;61(3):357-371. doi: 10.1007/s12020-018-1605-5.

31. Capoor MR, Nair D, Rajni, et al. Microflora of bile aspirates in patients with acute cholecystitis With or without cholelithi- 
asis: a tropical experience. Braz. J. Infect. Dis. 2008;12(3). doi: 10.1590/s1413-86702008000300012.

32. White JS, Hoper M, Parks RW, et al. Patterns of Bacterial Translocation in Experimental Biliary Obstruction. J. Surg. Res. 2006;132(1):80-84. doi: 10.1016/j.jss.2005.07.026.

33. Di Ciaula A, Garruti G, Baccetto RL, et al. Bile Acid Physiology. Ann. Hepatol. 2017;16(0):4-14. doi: 10.5604/01.3001.0010.5493.

34. Tsai CJ. Central adiposity, regional fat distribution, and the risk of cholecystectomy in women. Gut. 2006:55(5):708-714. doi: 10.1136/gut.2005.076133.

35. Tsai C-J, Leitzmann MF, Willett WC, Giovannucci EL. Prospective study of abdominal adiposity and gallstone disease in US men. Am J Clin Nutr. 2004;80(1):38-44. doi: 10.1093/ajcn/80.1.38

36. Nervi F, Miquel JF, Alvarez M, et al. Gallbladder disease is associated with insulin resistance in a high risk Hispanic population. J. Hepatol. 2006;45(2):299-305. doi: 10.1016/j.jhep.2006.01.026.

37. Mella JG, Schirin-Sokhan R, Rigotti A, et al. Genetic evidence that apolipoprotein E4 is not a relevant susceptibility factor for cholelithiasis in two high-risk populations. J. Lipid Res. 2007;48(6):1378-1385 doi: 10.1194/jlr.M700059-JLR200.

38. Yu K-j, Zhang J-r, Li Y, et al. Gallstone disease is associated with arterial stiffness progression. Hypertens. Res. 2016;40(1):31-34. doi: 10.1038/hr.2016.109

39. Sattar N, Gaw A, Scherbakova O, et al. Metabolic Syndrome With and Without C-Reactive Protein as a Predictor of Coronary Heart Disease and Diabetes in the West of Scotland Coronary Prevention Study. Circulation. 2003;108(4):414-419. doi: 10.1161/01.cir.0000080897.52664.94.

40. Mendez-Sanchez N, Bahena-Aponte J, Chavez-Tapia NC, et al. Strong Association between Gallstones and Cardiovascular Disease. Am J Gastroenterol. 2005;100(4):827-830. doi: 10.1111/j.1572-0241.2005.41214.x.

41. Koller T, Kollerova J, Hlavaty T, et al. Cholelithiasis and markers of nonalcoholic fatty liver disease in patients with metabolic risk factors. Scand. J. Gastroenterol. 2011:47(2):197-203. doi: 10.3109/00365521.2011.643481.

42. Arslan U, Turkoglu S, Balcioglu S, et al. Association between nonalcoholic fatty liver disease and coronary artery disease. Coron. Artery Dis. 2007:18(6):433-436. doi: 10.1097/MCA.0b013e3282583c0d.

43. Alberti KGMM, Eckel RH, Grundy SM, et al. Harmonizing the Metabolic Syndrome. Circulation. 2009;120(16):1640-1645. doi: 10.1161/circulationaha.109.192644

44. van der Wulp MYM, Verkade HJ, Groen AK. Regulation of cholesterol homeostasis. Mol. Cell. Endocrinol. 2013;368(1-2):1-16. doi: 10.1016/j.mce.2012.06.007.

45. Rader DJ, Alexander ET, Weibel GL, et al. The role of reverse cholesterol transport in animals and humans and relationship to atherosclerosis. J. Lipid Res. 2009;50(Supplement):S189-S194. doi: 10.1194/jlr.R800088-JLR200.

46. Jiang Z-Y, Parini P, Eggertsen $G$, et al. Increased expression of $L X$ Ra,ABCG5,ABCG8, andSR-Blin the liver from normolipidemic, nonobese Chinese gallstone patients. J. Lipid Res. 2008;49(2):464-472 doi: 10.1194/jlr.M700295-JLR200.

47. Schwartz C, Halloran L, Vlahcevic Z, et al. Preferential utilization of free cholesterol from high-density lipoproteins for biliary cholesterol secretion in man. Science. 1978;200(4337):62-64. doi: $10.1126 /$ science. 204996

48. Robins SJ, Fasulo JM. High density lipoproteins, but not other lipoproteins, provide a vehicle for sterol transport to bile. J. Clin. Invest. 1997;99(3):380-384. doi: 10.1172/jci119170.

49. Kim JM, Lee HL, Moon W, et al. Association between insulin, insulin resistance, and gallstone disease in Korean general population. Korean J Gastroenterol. 2007;50(3):183-187.

50. Shabanzadeh DM, Skaaby T, Sørensen LT, et al. Metabolic biomarkers and gallstone disease - a population-based study. Scand. J. Gastroen terol. 2017:52(11):1270-1277. doi: 10.1080/00365521.2017.1365166.

51. Wu Q, He X-D, Yu L, et al. The Metabolic Syndrome and Risk Factors for Biliary Tract Cancer: A Case-control Study in China. Asian Pac. J. Cancer Prev. 2012;13(5):1963-1969. doi: 10.7314/apjcp.2012.13.5.1963.

52. Su K, Sabeva NS, Liu J, et al. The ABCG5 ABCG8 Sterol Transporter Opposes the Development of Fatty Liver Disease and Loss of Glycemic Control Independently of Phytosterol Accumulation. J. Biol. Chem. 2012;287(34):28564-28575. doi: 10.1074/jbc.M112.360081.

53. Min H-K, Kapoor A, Fuchs M, et al. Increased Hepatic Synthesis and Dysregulation of Cholesterol Metabolism Is Associated with the Severity of Nonalcoholic Fatty Liver Disease. Cell Metab. 2012;15(5):665-674. doi: 10.1016/j.cmet.2012.04.004

54. Arrese M, Cortés V, Barrera F, Nervi F. Nonalcoholic fatty liver disease, cholesterol gallstones, and cholecystectomy. Current Opinion in Gastroenterology. 2018;34(2):90-96. doi: 10.1097/mog.0000000000000416.

55. Cortés V, Quezada N, Uribe S, et al. Effect of cholecystectomy on hepatic fat accumulation and insulin resistance in non-obese Hispanic patients: a pilot study. Lipids Health Dis. 2017;16(1). doi: 10.1186/s12944-017-0525-3.

56. Byrne CD, Targher G. NAFLD: A multisystem disease. J. Hepatol. 2015:62(1):S47-S64. doi: 10.1016/j.jhep.2014.12.012

57. Dietrich P, Hellerbrand C. Non-alcoholic fatty liver disease, obesity and the metabolic syndrome. Best Practice \& Research Clinical Gastroenterology. 2014;28(4):637-653. doi: 10.1016/j.bpg.2014.07.008.

58. Ahmed F, Baloch Q, Memon ZA, Ali I. An observational study on the association of nonalcoholic fatty liver disease and metabolic syndrome with gall stone disease requiring cholecystectomy. Annals of Medicine and Surgery. 2017;17:7-13. doi: 10.1016/j.amsu.2017.03.015.

59. Lammert F, Gurusamy K, Ko CW, et al. Gallstones. Nature Reviews Disease Primers. 2016;2:16024. doi: 10.1038/nrdp.2016.24.

\section{ИНФОРМАЦИЯ ОБ АВТОРАХ [AUTHORS INFO]}

*Осадчук Михаил Алексеевич, д.М.н., профессор [Michail A. Osadchuk, MD, PhD, Professor]; Aдpec: 119435, Mосква, ул. Большая Пироговская, д. 2, стр. 4 [Address: 2-4, Bolshaya Pirogovskaya street, Moscow, 119435, Russia]; eLibrary SPIN: 3108-0478, ORCID: https://orcid.org/0000-0003-1302-5832, e-mail: osadchuk.mikhail@yandex.ru.

Свистунов Андрей Алексеевич, д.м.н., проф., член-корреспондент PAH [Andrey A. Svistunov, MD, PhD, Professor], ; ORCID: https://orcid.org/0000-0003-1592-5703; e-mail: svistunov@sechenov.ru

Киреева Наталья Викторовна, К.М.Н., доцент [Natalia V. Kireeva, MD, PhD, Associate Professor], eLibrary SPIN: 68141680; ORCID: https://orcid.org/0000-0002-3467-886X, e-mail: kireeva_nat@inbox.ru

Осадчук Алексей Михайлович, д.м.н., профессор [Alexey M. Osadchuk, MD, PhD, Professor],

ORCID: https://orcid.org/0000-0002-8488-9235; eLibrary SPIN: 9455-3982; e-mail: maxlife2004@mail.ru

\section{ЦИТИРОВАТЬ:}

Свистунов А.А., Осадчук М.А., Киреева Н.В., Осадчук А.М. Желчнокаменная болезнь как клинический маркер метаболического синдрома// Ожирение и метаболизм. - 2018. - Т.15. — №. 3 - С. 3-8. doi: 10.14341/OMET9553

\section{TO CITE THIS ARTICLE:}

Svistunov AA, Osadchuk MA, Kireeva NV, Osadchuk AM. Gallstone disease as a clinical marker of metabolic syndrome. Obesity and metabolism. 2018;15(3):3-8. doi: 10.14341/OMET9553 